\title{
A Possible Difference in the Surface Relaxivity of Costal and Inland Sands
}

\author{
Joseph P. Hornak ${ }^{*}, 1$, Gianni Ferrante ${ }^{2}$, Andrew Coy $^{3}$ and Evan R. McCarney ${ }^{3}$ \\ ${ }^{I}$ Rochester Institute of Technology, Rochester, New York, USA; ${ }^{2}$ Stelar, Mede (PV), Italy; ${ }^{3}$ Magritek, Wellington, New \\ Zealand
}

\begin{abstract}
The ${ }^{1} \mathrm{H}$ nuclear magnetic resonance (NMR) spin-lattice relaxation rate of hydrated sands is related to the surface-to-volume ratio of the voids or pores between the hydrated sand grains and the surface relaxivity of the grains. The electron spin resonance (ESR) signal is often used to predict the relative surface relaxivity as the surface relaxivity is thought to be proportional to the concentration of paramagnetic species in the sand grains. We have identified a discrepancy in the surface relaxivity and ESR signal of an ocean beach sand compared to two sands of similar diameter from inland deposits. This difference can either be due to more surface weathering of the inland sand or more paramagnetic material from seawater adhering to the ocean sand.
\end{abstract}

Keywords: Hydrated sand, NMR surface relaxivity, spin-lattice relaxation rate, $\mathrm{R}_{1}$.

\section{INTRODUCTION}

The relationship between the ${ }^{1} \mathrm{H}$ nuclear magnetic resonance spin-lattice relaxation rate $\left(R_{I}\right)$ of a hydrated porous material, the $R_{I}$ of bulk water filling the pores $\left(R_{I B}\right)$, the surface relaxivity of the pores $(\rho)$, and the surface to volume ratio of the pores $(S / V)_{P}$ is the basis of many geophysical studies related to soil [1-3].

$\mathrm{R}_{1}=\mathrm{R}_{1 \mathrm{~B}}+\rho(\mathrm{S} / \mathrm{V})_{\mathrm{P}}$

NMR spin relaxation values are used to study hydration, porosity, and soil contamination [4-6]. Magnetic resonance sounding [7] is useful for finding aquifers and the $R_{1}$ value is often used to infer pore size. Near-surface magnetic resonance imaging $[8,9]$ of hydrated soil may one day allow imaging of buried utilities. The signal from this technique is strongly dependent on the $R_{l}$ value of the hydrated soil.

We recently reported on this relationship for some fully hydrated unconsolidated natural and synthetic sands [4]. In that study we adopted the convention that for similarly shaped grains, $(S / V)_{P}$ is inversely proportional to the sand grain diameter $(d)$. In doing so, we were able to fit Eq. (1) to $R_{l}$ values for different diameter sands, thus calculate $\rho$ for the sands. The $\rho$ values used for the fit were proportional to the electron spin resonance signal for the dry sands. This is a reasonable find as the surface relaxivity is caused by paramagnetic impurities in the surface layer of atoms of the grains. The concentration of paramagnetic impurities in the entire grain volume, represented by the ESR signal, is presumed to be uniform throughout and the same as the concentration in the outer surface layer governing $\rho$.

A preliminary report indicated that this relationship may not be true for all sands [10]. Surface weathering or surface adsorption of paramagnetic metals may cause the concentra-

*Address correspondence to this author at the RIT Magnetic Resonance laboratory, Center for Imaging Science, Rochester Institute of Technology, 54 Lomb Memorial Drive, Rochester, NY 14623-5604, USA; Tel: 585.475.2904; Fax: 585.475.5988; E-mail: jphsch@rit.edu tion of these metals on the surface layer to differ from that within the grain. The comparison made to exemplify this difference is between an active beach sand from Asilomar, CA USA, and two inland sands from Illinois USA. We present the details of these findings in this paper.

In the previous paper, we reported $R_{1}$ values at proton resonance frequencies $(v)$ between $30 \mathrm{MHz}$ and $10 \mathrm{kHz}$. This data was used to extrapolate a value for $R_{l}$ and $\rho$ at $v=2.5$ $\mathrm{kHz}$, the approximate resonance frequency of protons in the magnetic field of the Earth. This extrapolation was risky because the dispersion in $R_{I}$ with $v$ could either continue at $v$ $<10 \mathrm{kHz}$ or stop and $R_{l}$ level off. In the current presentation, we have added a datum point at $1.9 \mathrm{kHz}$ using an Earth's field NMR spectrometer that eliminated the need to extrapolate.

\section{BACKGROUND}

The $R_{I}$ of a nuclear spin system is influenced by timevarying magnetic fields. In pure water, $R_{l}$ results from dipole-dipole interactions between water protons modulated by the rotational motions. The frequency dependence of these time varying magnetic fields is given by the spectral density function, $J(v)$, for bulk water [11]. $J(v)$ is flat over a broad range of frequencies from zero to a frequency equal to the inverse of the correlation time $\left(\tau_{c}\right)$ for the rotational motion, where $J(v)$ decreases to zero. This change in $J(v)$ is referred to as a dispersion and plots of the $R_{l}$ value as a function of $v$ are referred to as NMR dispersion plots. The $R_{I}$ of the ${ }^{1} \mathrm{H}$ spins in pure water depends on the number of time-varying magnetic fields at $v$ and $2 v$ experienced by the nucleus [12]. Water molecules experiencing an electrical charge from ions will possess a different $\tau_{\mathrm{c}}$ due to the electrostatic attraction between the polar water molecule and the ion.

In the presence of paramagnetic ions, the water $R_{l}$ is also influenced by electron-nuclear dipolar and contact interactions between the nuclear spin of water hydrogens and the electron spin of paramagnetic material $[13,14]$. The correlation times for these interactions are influenced by a rotational correlation time for a water molecule in the hydration 
sphere of the paramagnetic material, the electron spin-lattice relaxation time, the proton-electron hyperfine coupling constant, and the electron spin-spin relaxation time.

The $R_{l}$ of water adsorbed on a surface is also influenced by electron-nuclear dipolar and contact interactions, except the interaction is between the nuclear spin of water and the electron spin of paramagnetic material both in and on the surface. These interactions are influenced by the correlation times described previously for paramagnetic ions in solution, plus ones for diffusion $\left(\tau_{\mathrm{m}}\right)$ and desorption $\left(\tau_{\mathrm{s}}\right)$ motions of the water on and from the surface of a particle [15].

Randomly packed, unconsolidated, solid sand particles form a network of pores connected by channels [16]. The size of a pore is proportional to the diameter of the particles [15]. When fully hydrated, water fills the pores and channels between the particles. The mechanism of NMR spin relaxation in porous media is well established [1-3], and can be divided into two limiting cases: fast-diffusion or surfacelimited, and slow-diffusion or diffusion-limited. In the fastdiffusion case, the magnetization recovery for a single pore is monoexponential and depends on the $(\mathrm{S} / \mathrm{V})_{\mathrm{P}}$. In the slowdiffusion regime, the dominant relaxation occurs at the surface but the diffusion of spins to the surface is slow. In this case for a single pore, the return of magnetization to equilibrium is multiexponential and depends on the pore shape. McCall et al. [17] have presented modifications to the theory for coupled-pore systems. Sands similar to the Asilomar sand contain well-coupled pores of similar dimensions [4] and hence we focus on the fast-diffusion case.

In the fast-diffusion case, $R_{l}$ is influenced by bulk water and a thickness of water $(\lambda)$ adsorbed on the surface of the pore, both with distinctly different $R_{l}$ values. On the timescale of the NMR relaxation measurement, water molecules readily diffuse between the two environments. $R_{l}$ is a function of $R_{1_{B}}$ and the surface relaxation rate $\left(R_{1_{S}}\right)$.

$$
R_{1}=R_{1_{B}}+\lambda R_{1_{S}}\left(\frac{S}{V}\right)_{P}
$$

Eqn. 2 is an alternative representation of eqn. 1 as the surface relaxivity is defined as $\lambda R_{1_{S}}$.

Similar to the relaxation in porous materials model of eqns. 1 and 2, water in the presence of solvated diamagnetic ions can be characterized as existing in two different environments. There is structured water in the solvation shell of the ions, and bulk water not experiencing the effect of the ions. The fraction of structured water associated with the salvation shells of the ions is $\phi$, and the remaining fraction $(1-\phi)$ is bulk water. On the NMR timescale, with rapid exchange between the two environments the measured relaxation rate for an aqueous solution of diamagnetic ions becomes

$$
R_{1}=(1-\phi) R_{1_{B}}+\phi R_{1_{H}}
$$

where $R_{1_{H}}$ is the $\mathrm{R}_{1}$ of the structured water in the hydration shell $[18,19]$. $R_{1_{H}}$ is characterized by a unique $\tau_{\mathrm{c}}$ from dipole-dipole interactions between the water molecules in the hydration shell.
In the fast-diffusion case, the $J(v)$ from all the above interactions contribute, although not equally, to the overall frequency dependence of $R_{l}$ for hydrated sands. Fig. (1) summarizes the frequency dependence of these interactions. For bulk water systems at $20{ }^{\circ} \mathrm{C}$, the rotational $\tau_{\mathrm{c}} \approx 3.5 \times 10^{-12} \mathrm{~s}$ [11], placing the dispersion from these motions at a frequency well beyond the reach of NMR spectrometers. The interaction of water protons with paramagnetic ions in solution or in the lattice can cause dispersions between approximately $10^{5}$ and $10^{11} \mathrm{~s}^{-1}$. The surface diffusion/desorption interaction is unique in that it is characterized by a large constant $R_{l}$ at $v<1 / \tau_{\mathrm{s}}$, a small constant $R_{l}$ at $v>1 / \tau_{\mathrm{m}}$, and a logarithmic relation between $1 / \tau_{\mathrm{s}}$ and $1 / \tau_{\mathrm{m}}$. The value of $\tau_{\mathrm{m}}$ is $\sim 4 \times 10^{-10} \mathrm{~s}$ and $\tau_{\mathrm{s}}$ is less than $\sim 1 \times 10^{-4} \mathrm{~s}[15]$. The surface diffusion/desorption interaction is also unique in that it produces a dispersion with a linear relationship between $R_{l}$ and $\log (\mathrm{v})$ while the other interactions all produce power law dispersions.

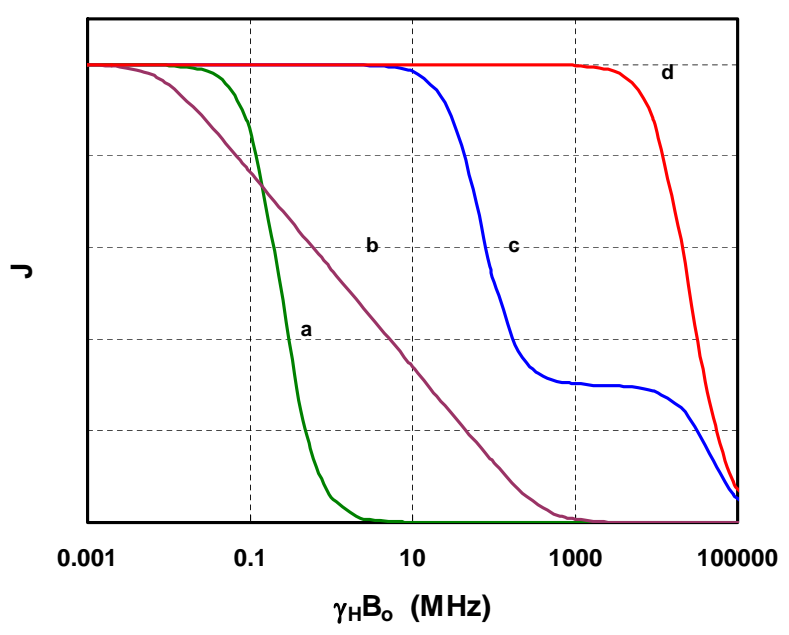

Fig. (1). Plots of the normalized spectral density function $(\mathrm{J})$ as a function of magnetic field reported as proton NMR frequency for a) proton-electron hyperfine, b) surface diffusion/desorption, c) proton-electron dipolar, and d) proton-proton dipolar interactions.

The surface relaxivity is caused by the electron-nuclear dipolar and contact interactions between the ${ }^{1} \mathrm{H}$ nuclear spins in water and paramagnetic materials on and in the sand grains. These paramagnetic substances can be either adsorbed on the surface [20], or randomly distributed throughout the grain and hence exposed at the surface. With the latter, it is possible to assess the surface concentration using ESR as the area of the ESR absorption signal is proportional to the concentration of electron spins in the sample. For paramagnetic materials adsorbed on the surface, it is only possible to relate the ESR signal to $\rho$ when the concentration of spins in the grains is known separately or zero.

\section{MATERIALS AND METHODOLOGY}

Three natural quartz sands were compared: Asilomar Beach, Ottawa, and Oregon Sands. The first is from the Asilomar Beach in Asilomar, CA USA and these sand grains are currently being formed from the erosion of the native bedrock. Both the Ottawa and Oregon sand grains were formed 
from the same erosive forces associated with a Paleozoic era sea approximately 465 million years ago [21]. Since then, these two sands have experienced varying degrees of surface weathering.

The Asilomar Beach Sand was collected fully hydrated with ocean water and used without sieving. This fully hydrated sand was used as is for the NMR studies. For ESR and geometric measurements, the sand was rinsed with 18 $\mathrm{M} \Omega \cdot \mathrm{cm}$ deionized (DI) water and dried at $200{ }^{\circ} \mathrm{C}$. Ottawa Sand (F110, US Silica, Berkeley Springs, WV, USA) and Oregon Sand (7020 Granusil, Unimin Corp., New Canaan, CT, USA) were acquired from the respective suppliers. The Ottawa and Oregon sand samples were cleaned by rinsing with DI water, oven dried at $200{ }^{\circ} \mathrm{C}$, and sieved using a mechanical $76 \mathrm{~mm}$ sieve shaker (SS-5, Gilson Co. Inc., Worthington, $\mathrm{OH}$ ) to obtain the diameters studied [4]. ESR and geometric measurements were made on the dry samples, while NMR was performed on the sands fully hydrated with DI water.

The geometric properties of all the sand grains were measured using an optical microscope (Eclipse E600PL, Nikon) with image analysis software (analySIS, Olympus). The average minimum and maximum diameters $\left(d_{\min }\right.$ and $d_{\max }$ ) of a random sampling of 100 grains were measured and used to calculate the aspect ratio $\left(R_{A}=d_{\max } / d_{\min }\right)$, the average diameter, and uniformity $\left(\sigma_{d} / d\right)$ of the grains. All the geometric properties are used to estimate similarities in $(S / V)_{P}$.

The $R_{l}$ values of the Asilomar Sand were measured by two techniques. $R_{I}$ values were measured at an Earth's magnetic field of $44.6 \mu \mathrm{T}(1.9 \mathrm{kHz})$ using an Earth's field NMR spectrometer (Terranova-MRI, Magritek, Wellington, New Zealand). This same instrument was used to measure the $R_{l}$ in its polarizing field of $18 \mathrm{mT}(766 \mathrm{kHz})$. Both of these measurements were made on $250 \mathrm{ml}$ volume samples. $R_{l}$ values as a function of field strength were measured between $0.47 \mathrm{mT}(20 \mathrm{kHz})$ and $0.19 \mathrm{~T}(8 \mathrm{MHz})$ using a field cycling NMR spectrometer (SMARtracer, Stelar, Mede, Italy). Field cycling measurements were made on samples in $10 \mathrm{~mm}$ NMR tubes. The $R_{l}$ value for the Asilomar sand was interpolated at $2.5 \mathrm{kHz}$ from Fig. (2).

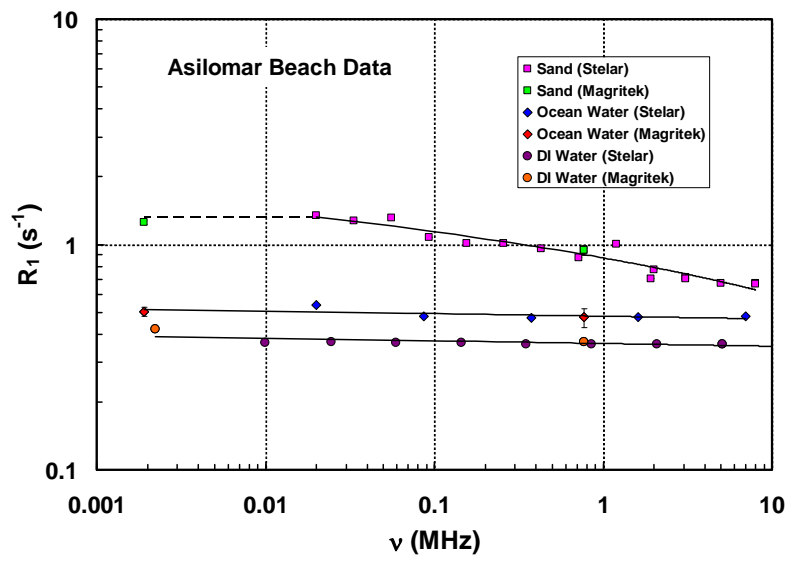

Fig. (2). ${ }^{1} \mathrm{H}$ NMR $\mathrm{R}_{1}$ values as a function of resonance frequency between $1.9 \mathrm{kHz}$ and $9 \mathrm{MHz}$ for fully hydrated Asilomar Beach sand, Asilomar Beach ocean water, and deionized (DI) water as measured by an Earth's field (Magritek) and field cycling (Stelar) NMR spectrometers.
$R_{I}$ values for the Ottawa and Oregon Sands were taken from [4]. This study measured $R_{l}$ as a function of $d$ and $v$. Because of the high density of data points in $d$ from this study, our $R_{l}$ values for these two sands were calculated from their fitted plots of $R_{l}$ vs. $d$ at $d=340 \mu \mathrm{m}$, and thus assuring comparison values for similar diameter sand grains.

The paramagnetic metal content of the sand was determined using a low frequency electron spin resonance ESR spectrometer [22] operating at $247 \mathrm{MHz}$ with a magnetic field sweep between 0 and $15 \mathrm{mT}$. Sand samples were prepared by rinsing with $18 \mathrm{M} \Omega \cdot \mathrm{cm}$ deionized water and dried. Sand samples were placed in $2 \mathrm{~cm}$ diameter glass sample tubes to a height that completely filled the ESR sample probe. Conventional first-derivative ESR spectra were recorded. The relative number of paramagnetic spins per mass of sand $\left(N_{m}\right)$ was calculated from the double integral of the ESR spectrum $(S)$ minus the same for an empty sample tube $\left(S_{o}\right)$ all divided by the mass of sand filling the probe $(\mathrm{m})$.

$\mathrm{N}_{\mathrm{m}}=\left(\mathrm{S}-\mathrm{S}_{\mathrm{o}}\right) / \mathrm{m}$

Assuming similar density quartz sand grains, this $N_{m}$ is proportional to the concentration of spins in a grain.

\section{RESULTS AND DISCUSSION}

All three sands had similar aspect ratios and mean diameters. (See Table 1). Based on this, the sand grains should pack similarly and have similar $(S / V)_{P}$ ratios. However, the distribution of grain diameters in the Asilomar Sand was greater than that for the Ottawa and Oregon Sand samples, because the Asilomar Sand was not sieved. This 2.3 times larger distribution of $d$ will cause a difference in the packing and $(S / V)_{P}$, which we will address later.

Table 1. Comparison of Hydrated Sands at $v=2.5 \mathrm{kHz}$

\begin{tabular}{|l|l|l|l|}
\hline Property & Asilomar & Ottawa $^{\mathrm{a}}$ & Oregon $^{\mathrm{a}}$ \\
\hline \hline$d(\mu \mathrm{m})$ & 340 & 340 & 340 \\
\hline$\sigma_{d} / d$ & 0.30 & 0.13 & 0.13 \\
\hline$R_{A}$ & 1.5 & 1.5 & 1.4 \\
\hline Relative ESR Signal & 0.9 & 1.0 & 1.7 \\
\hline Relative $\rho$ & 2.5 & 1.0 & 1.6 \\
\hline$\left(R_{I}-R_{I B}\right)\left(\mathrm{s}^{-1}\right)$ & 0.80 & 0.32 & 0.49 \\
\hline
\end{tabular}

${ }^{\mathrm{a}}$ Data at specified diameter were interpolated from [4].

The $R_{I}$ values for Asilomar Beach sand hydrated with ocean water, Asilomar Beach ocean water, and DI water as a function of proton resonance frequency are presented in Fig. (2). Both Stelar and Magritek instruments produced consistent data at their overlapping frequency of $766 \mathrm{kHz}$. Compared to $18 \mathrm{M} \Omega \cdot \mathrm{cm}$ deionized water, the ocean water shows a higher $R_{l}$ at all $v$ values. This is attributable the $0.5 \mathrm{M}$ diamagnetic salt ions in sea water acting through eqn. 3 , and not the $<30 \mathrm{nM}$ paramagnetic ions [23]. The slight slope of the line for the two bulk water samples is not predicted by theory and may reflect measurement uncertainty.

The $R_{l}$ of the hydrated sand grains was greater than that for ocean water and increased with decreasing resonance 
frequency to about $20 \mathrm{kHz}$. The dispersion in $R_{l}$ between 20 $\mathrm{kHz}$ and $8 \mathrm{MHz}$ is the result of surface relaxation due to interactions of the water with paramagnetic impurities in the sand grains and is consistent with theory [15]. It was not possible to determine if the data followed a power law or $\log (v)$ relationship as both forms fit the data equally well. Below $20 \mathrm{kHz}, R_{l}$ appears to level off. The leveling off is significant because it sets a lower limit on the dispersion. Extrapolation of the dispersion to the resonance frequency of ${ }^{1} \mathrm{H}$ in the Earth's magnetic field may yield a higher $R_{l}$. It may be more appropriate to use the $R_{l}$ value at lowest frequency of the field cycling studies, i.e. 10 or $20 \mathrm{kHz}$, as the extrapolated $R_{l}$ at $2.5 \mathrm{kHz}$ then to extend the dispersion beyond the $10 \mathrm{kHz} R_{l}$ value. This hints that the extrapolation in the previous study may have produced slightly higher values at $2.5 \mathrm{kHz}$ then actual values.

The $R_{l}$ value of the Asilomar Sand was compared to that of the previously reported Ottawa and Oregon, IL Sands [4]. Fig. (3) shows a plot of $\left(R_{l}-R_{l B}\right)$ vs. $\log (d)$ for the Ottawa and Oregon Sands. The data was fit with Eq. (1) to obtain the relative $\rho$ values of 1.0 and 1.6 respectively. (See Table 1). These $\rho$ values were proportional to the relative ESR signals of 1.0 and 1.7. The $R_{l}$ values were measured for several different diameter samples; however, these were not the same as the Asilomar Sand grain diameter. Therefore, the $\left(R_{l}-R_{l B}\right)$ values of Table 1 are based on the fit to the data in Fig. (3) at $d=340 \mu \mathrm{m}$. For comparison purposes, the datum point for the Asilomar Sand in Fig. (3) is displayed with a line segment for a $\rho=2.5$. This relative $\rho$ value was determined by fitting eqn. 1 to the point.

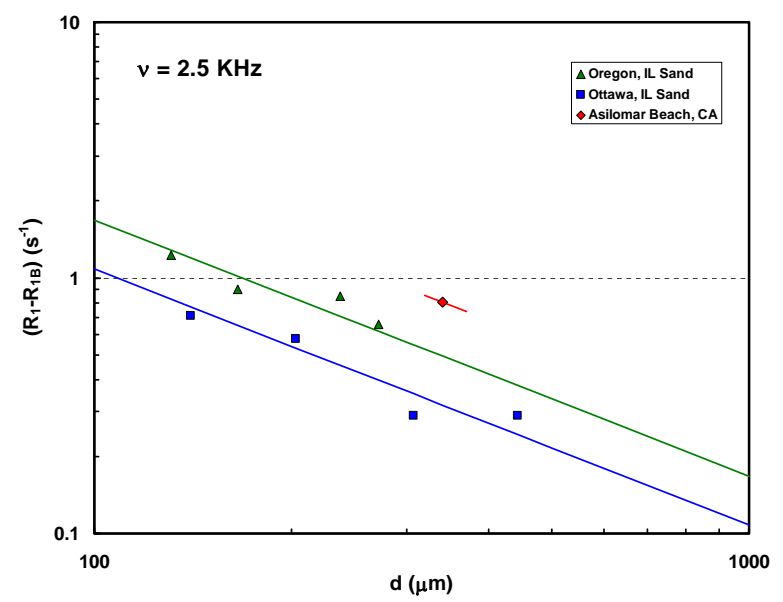

Fig. (3). ${ }^{1} \mathrm{H}$ NMR $\left(\mathrm{R}_{1}-\mathrm{R}_{1 \mathrm{~B}}\right)$ values as a function of diameter (d) for fully hydrated Oregon $(\bullet)$, Ottawa $(\square)$, and Asilomar Beach $(\diamond)$ Sands. Solid lines are best fits of the data using Eq. (1). The line segment through the Asilomar Sand point is provided as a reference for the $\rho$ value.

The previous study of hydrated Ottawa and Oregon sands showed that the measured $\left(R_{1}-R_{l B}\right)$ values correlated well with the ESR signal and could thus be used to predict $\rho$ [4]. In our study, the $\left(R_{1}-R_{1 B}\right)$ value of hydrated Asilomar Sand is greater than that of inland Ottawa and Oregon Sands. (See Table 1). As per Eq. (1), the difference must be due to $(S / V)_{P}$ or $\rho$. The similar $d$ and $R_{A}$ values would assure very similar packing of the grains if the $\sigma_{d} / d$ values were similar. The literature suggests that the difference in $d$ must be greater than 6.5 to see a significant change in the porosity [24,25]. Therefore, the 2.3 times larger $\sigma_{d}$ may not cause a significant change in $(S / V)_{P}$ and is not the primary cause of the larger $\left(R_{l}-R_{l B}\right)$ for Asilomar Sand. This points to $\rho$ as the cause of the larger $\left(R_{1}-R_{1 B}\right)$ for the Asilomar Sand.

The ESR signal of the Ottawa and Oregon Sands correlate well with the $\rho$ value needed to fit the data of Fig. (3), but the Asilomar Sand $\rho$ value does not. This observation leads us to believe that the concentration of paramagnetic material on the surface of the grains of sand may be different from the concentration in the center. The ESR signal is proportional to the total paramagnetic concentration. Either a higher surface concentration of paramagnetic material in the Asilomar Sand, or a lower surface concentration of paramagnetic material in the Ottawa and Oregon Sands could account for the difference. The former could be attributed to paramagnetic material from the seawater adhering to the surface of the grains. The rinsing of the sand for ESR measurements and the ESR averaging of the signal from the surface and interior of the grains would account for the higher $\rho$ than predicted by ESR. The latter cause could come about from repeated weathering of the surface of the inland sand grains. Acid washing the surface of the Asilomar Sands might remove the adsorbed and surface layer paramagnetic materials, however rehydrating with ocean water might cause some ions to be readsorbed. The best way to distinguish between the two causes is to use an analytical technique that measures only the surface concentration of these trace paramagnetic metals.

\section{CONCLUSIONS}

Unlike in a previous study where the ESR signal was found to be proportional to the surface relaxivity of several sands and glass spheres, in this study the ESR signal was found not to be proportional to the surface relaxivity of a specific ocean beach sand hydrated with ocean water. Although $(S / V)_{P}$ could not be completely ruled out as the cause, it is believed that a difference between the surface and overall concentration of paramagnetic material for the sand grains is most probably the cause. The difference may be due to paramagnetic ions found in sea water being adsorbed on the surface of the grains of Asilomar Sand, or the weathering of paramagnetic ions out of the surface layer in the geologically older inland sands. More studies are needed to confirm the cause and determine the best analytical technique for measuring the surface relaxivity.

\section{REFERENCES}

[1] Korringa J, Seevers DO, Torrey HC. Theory of spin pumping and relaxation in systems with low concentration of electron spin resonance centers. Phys Rev 1962; 127: 1143-50.

[2] Brownstein KR, Tarr C. Importance of classical diffusion in NMR studies of water in biological cells. Phys Rev A 1979; 19: 2246453.

[3] Kleinberg RL, Kenyon WE, Mitra PP. Mechanism of NMR relaxation of fluids in rock. J Magn Reson A 1994; 108: 206-14.

[4] Bray CL, Bryant RG, Cox MJ, et al. The ${ }^{1} \mathrm{H}$ nuclear magnetic resonance spin-lattice relaxation rate of some hydrated synthetic and natural sands. J Environ Eng Geophys 2009; 14: 49-61.

[5] Hertzog RC, White TA, Straley C. Using NMR decay time measurements to monitor and characterize DNAPL and moisture in subsurface porous media. J Environ Eng Geophys 2007; 4: 293-306. 
[6] Pohlmeier A, Haber-Pohlmeier S, Stapf S. A fast field cycling nuclear magnetic resonance relaxometry study of natural soils. Vadose Zone J 2009; 8: 735-42.

[7] Legchenko A, Baltassat J-M, Beauce A, Bernard J. Nuclear magnetic resonance as a geophysical tool for hydrogeologists. J Appl Geophys 2002; 50: 21-46.

[8] Bray CL, Hornak JP. Unilateral MRI using a rastered projection. J Magn Reson 2007; 188: 151-9.

[9] Bray CL, Hornak JP. Underground Variations in $B_{\text {Earth: Implica- }}$ tions for Near-Surface MRI. Concepts Magn Reson 2009; 35B: 153-67.

[10] Hornak JP, Ferrante G, Coy A, McCarney ER. A ${ }^{1}$ H NMR SpinLattice Relaxation Time Study of Asilomar Sands. In: $50^{\text {th }}$ Experimental NMR Conference, 2009; Asilomar, CA, USA.

[11] Bloembergen N, Purcell EM, Pound RV. Relaxation effects in nuclear magnetic resonance absorption. Phys Rev 1948; 73: 679712 .

[12] Abragam A. The principles of nuclear magnetism. Clarendon: Oxford 1961.

[13] Soloman I. Relaxation process in a system of two spins. Phys Rev 1955; 99: 559-65.

[14] Bakhmutov VI. Practical NMR relaxation for chemists. Wiley: Hoboken, NJ 2004.

[15] Godefroy S, Korb J-P, Fleury M, Bryant RG. Surface nuclear magnetic relaxation and dynamics of water \& oil in macroporous media. Phys Rev E 2001; 64: (021605)1-13.
[16] Hills BP, Belton PS, Quantin VM. Water proton relaxation in heterogeneous systems: I. Saturated randomly packed suspensions of impenetrable particles. Mol Phys 1993; 78: 893-908.

[17] McCall KR, Johnson DL, Guyer RA. Magnetization evolution in connected pore systems. Phys Rev B 1991; 44: 7344-55.

[18] Broersma S. Nuclear magnetic relaxation of nonuniform systems. J Chem Phys 1956; 24: 153-60.

[19] Melnichenko NA, Bazhanov AV, Kupriyanov AS. Temperature dependence of NMR relaxation rate in some aqueous electrolytes of semimolar concentration. J Struct Chem 2003; 44: 397-403.

[20] Bryar TR, Daughney CJ, Knight RJ. Paramagnetic effects of iron(III) species on nuclear magnetic relaxation of fluid protons in porous media. J Magn Reson 2000; 142: 74-85.

[21] Pitman JK, Goldhaber MB, Spöetl C. Regional diagenetic patterns in the St. Peter Sandstone: implications for brine migration in the illinois basin. U.S. Geological Survey Bulletin 2094-A, United States Government Printing Office, Washington, DC USA 1997.

[22] Hornak JP, Spacher M, Bryant RG. A modular low frequency ESR spectrometer. Meas Sci Technol 1991; 2: 520-22.

[23] Millero FJ. Chemical oceanography. Boca Raton: Taylor \& Francis 2006; pp. 91-2.

[24] Cubrinovski M, Ishihara K. Maximum and minimum void ratio characteristics of sands. Soils Found 2002; 42: 65-78.

[25] Westman ER, Hugill HR. The packing of particles. J Am Ceram Soc $1930 ; 13: 767-79$.

(C) Hornak et al.; Licensee Bentham Open.

This is an open access article licensed under the terms of the Creative Commons Attribution Non-Commercial License (http://creativecommons.org/licenses/by-nc/3.0/) which permits unrestricted, non-commercial use, distribution and reproduction in any medium, provided the work is properly cited. 